\title{
Elecciones al Parlamento catalán 2012-2017:
}

\section{¿un proceso acelerado de realineamiento \\ electoral?}

Elections to the Catalan Parliament 2012-2017: An accelerated process of electoral realignment?

Cómo citar

este artículo en APA: Serra Serra, M., Ubasart-González, G. y Marti i Puig, S. (2020). Elecciones al Parlamento catalán 2012-2017: ¿un proceso acelerado de realineamiento electoral? Analecta Política, 10(18), 105-124.

Fecha de recepción: 26.03.2020

\section{MACIÀ SERRA SERRA}

Doctor en Ciencia Política

Universitat de Girona, Espańa

Correo electrónico: Macia.serra@udg.edu

\section{GEMMA UBASART-GONZÁLEZ}

Doctora en Ciencia Política

Universitat de Girona, España gemma.ubasart@udg.edu

\section{SALVADOR MARTÍ I PUIG}

Doctor en Ciencia Política

Universitat de Girona, Espańa Correo electrónico: salvador.marti@udg.edu 


\section{Resumen \\ El artículo analiza las dinámicas de competencia electoral que se desarrollan en Cataluña entre 2012 y 2017 para validar la hipótesis de que el sistema de partidos catalán cambia su lógica de competencia fruto de un proceso de realineamiento electoral. Se revisan las pautas de comportamiento electoral y la estructura del sistema de partidos en Cataluña desde las primeras elecciones después de la recuperación de la Generalitat (1980) hasta 2017, así como los estudios más relevantes sobre la cuestión. Desde 2012, ha aumentado la fragmentación partidista y la competencia; antes centrípeta, se ha convertido en centrífuga, a la vez que la disputa alrededor de la cuestión nacional se ha impuesto sobre el eje ideológico. El marco analítico utilizado es el del realineamiento electoral y la metodología es la comparación diacrónica a partir de una descripción densa de caso. En las conclusiones, se afirma que en Cataluña se ha llevado a cabo un acelerado proceso de realineamiento electoral. Los seis elementos que la literatura señala aparecen en el caso: una gran participación electoral, la aparición de conflictos en la nominación de candidatos, la irrupción de "terceros" partidos, el incremento de volatilidad, la aparición de nuevos temas de conflicto y un aumento de la polarización político-ideológica.}

Palabras clave: Cataluña, elecciones críticas, sistema de partidos, realineamiento, independentismo.

\section{Abstract}

The article analyzes the dynamics of electoral competition that take place in Catalonia between 2012 and 2017. The objective is to validate the hypothesis that the Catalan party system changes its logic of competition as a result of an electoral realignment process. The electoral behavior guidelines and the structure of the party system in Catalonia are reviewed from the first elections after the recovery of the Generalitat (1980) until 2017. Also, the most relevant studies on the matter are reviewed. Since 2012, partisan fragmentation and competition have increased. It has gone from being centripetal to centrifugal. At the same time, the dispute around the national matter has been imposed on the ideological axis. The analytical framework used is that of electoral realignment and the methodology is the diachronic comparison based on a dense case description. In the conclusions, it is stated that an accelerated electoral realignment process has been carried out in Catalonia. The six elements indicated by the literature appear in the case: a large electoral participation, the appearance of conflicts in the nomination of candidates, the emergence of "third" parties, the increase in volatility, the appearance of new issues of conflict and an increase of political-ideological polarization.

Keywords: Catalonia, critical elections, party system, realignment, pro-independence movement. 


\section{Introducción}

El 11 de septiembre de 2012 las calles de Barcelona albergaban una gran manifestación independentista. Si bien la celebración del día nacional de Cataluña siempre ha sido una cita reivindicativa, en este caso se daban dos novedades: la movilización fue masiva y tenía un carácter secesionista. La cuestión nacional ha estado presente en la vida política catalana y ha tenido un importante protagonismo en la vida española desde la segunda mitad del siglo XIX, pero el independentismo siempre había sido una corriente minoritaria (Amat, 2017). Este evento inicia un intenso periodo de movilización social, política e institucional que acaba acompańando a importantes transformaciones en la cultura política, el sistema de actores políticos y los patrones de comportamiento electoral. Las mutaciones también acaban permeando la vida política española (Ubasart-González, 2020).

Este artículo quiere analizar una arista de estas transformaciones, esto es, las modificaciones que se producen en el sistema de partidos y las pautas de comportamiento electoral. Se pone el foco en los distintos eventos electorales para elegir el legislativo catalán (el Parlament) que se han producido durante esta etapa definida popularmente como procés independentista. Esta empieza, como se ha apuntado, en septiembre de 2012 y se cierra en las elecciones que se celebran después de los "hechos de octubre" de 2017 (votaciones el 1-O, declaración formal de independencia, suspensión de la autonomía y encarcelamiento de líderes políticos y sociales).

En concreto, este trabajo se pregunta si en el periodo que va desde las elecciones anticipadas al Parlamento catalán del 25 de noviembre de 2012 hasta las del 21 de diciembre de 2017 se lleva a cabo un proceso de realineamiento electoral. Los textos seminales sobre el tema exponen que este fenómeno sucede cuando en unas elecciones se produce una súbita alteración del clivaje preexistente entre el electorado y aparece uno nuevo o se modifica el anterior, y se genera una nueva articulación del sistema de partidos y las dinámicas de competencia de este (Key, 1955, 1964).

Los resultados de las elecciones del 21 de diciembre de 2017 tienen muy poco que ver con los que se podían observar en el Parlament en tiempo del "oasis" pujolista (1980-2003) ${ }^{1}$ o de los gobiernos catalanistas y de izquierdas de Maragall y

1 Este concepto se utiliza para caracterizar un periodo de gobierno, pero también de gobernabilidad social y cultural, caracterizado por la hegemonía del centro derecha catalanista representado en las siglas de Convergencia i Unió (CiU) y de su líder Jordi Pujol. 


\section{8 | Macià Serra Serra, Gemma Ubasart-González \\ ¿un proceso acelerado de realineamiento electoral?}

Montilla (2003-2010). ${ }^{2}$ Si se observan con detalle los resultados de las elecciones catalanas desde 2012, estos dan cuenta que el marco de competencia electoral se ha transformado de manera rápida: ha aumentado la fragmentación y la competencia centrífuga, a la vez que la competencia sobre la cuestión nacional (a pesar de estar presente en la vida política catalana desde la segunda mitad del siglo XIX) se ha impuesto sobre la competencia en el eje ideológico izquierda-derecha (que hasta la fecha se presentaba como eje central). Es más, durante el lustro 20122017 ha saltado por los aires el consenso catalanista, tanto en el ámbito partidista como en la opinión pública y publicada (Guibernau, 2004).

El artículo se divide en tres apartados. El primero expone unos apuntes teóricos y metodológicos alrededor de la literatura sobre realineamiento electoral que destacan aquellas cuestiones de interés para el trabajo. El segundo hace referencia a la dinámica tradicional de la competencia electoral en Cataluña (1984-2010) y la irrupción de un nuevo escenario en 2012. Finalmente, el tercero y último analiza si en Cataluña se han dado los elementos que los teóricos señalan como condición para que se pueda hablar de la existencia de un proceso de realineamiento electoral.

\section{Apuntes teóricos y metodológicos}

El estudio del realineamiento electoral se ha caracterizado, fundamentalmente, por analizar ciclos temporales prolongados con la intención de observar e identificar coyunturas específicas que suponen cambios de larga duración respecto de la dinámica de competencia política entre partidos. Quienes han trabajado sobre procesos de realineamiento señalan que este se da si concurren seis elementos, a saber, la existencia de una gran participación electoral, la aparición de conflictos en la nominación de candidatos dentro de alguno de los grandes partidos en liza, la irrupción de "terceros partidos" en la arena electoral, la aparición de nuevos temas de conflicto (issue cleavages), una relevante polarización político-ideológica y, finalmente, cambios sustanciales en las propuestas de políticas públicas (Mayhew, 2002).

2 Maragall y Montilla son miembros del Partit dels Socialistes de Catalunya (PSC), partido asociado al Partidos Socialista Obrero Español (PSOE), del que es referente en Cataluña. 
En caso de darse estos elementos o una porción importante de ellos, se podría afirmar que en Cataluña se celebraron unas elecciones críticas (o una sucesión de elecciones) que desencadenaron, en un periodo relativamente breve, cambios sustanciales en la dinámica electoral y en el sistema de partidos del país. De ser así, podría afirmarse que ha existido un realineamiento electoral.

Las transformaciones operadas en el sistema de partidos y el marco de competencia electoral no son una especificidad propia catalana. En Europa Occidental, son muchos los países en los que, a partir de la irrupción de la crisis económica de 2008 (que también deriva en política y cultural), surgen nuevos partidos (Fernández-Albertos, 2018; Hernández \& Kriesi, 2016; Kriesi \& Pappas, 2015), entran en crisis algunos de los partidos tradicionales, cambian los ejes principales de debate, muta la magnitud de voto en las fuerzas políticas, etc. El aumento de la fragmentación, la volatilidad y la polarización se encuentra en varios de los marcos de competencia electoral de los países vecinos. Ahora bien, en algunos de estos casos, se produce un proceso de realineamiento electoral, pero en otros se mantiene la misma estructura de competición con más actores o con actores. En el caso catalán afirmamos que sí.

Para analizar lo acontecido, es necesario señalar que, aunque el sistema de partidos catalán tiene una relativa autonomía y especificidad, "estamos frente de sistemas de partidos [el catalán y el español] interrelacionados y de ninguna manera aislados" (Baras y Matas, 1998, p. 185). Las influencias entre ambos sistemas de partidos son mutuas, se relacionan y condicionan (Marcet, 2011). Las especificidades del subsistema catalán tendrían que ver con un sistema de ejes propio, ya que coexiste el eje nacional y el ideológico (Molas y Bartomeus, 1998), la existencia de partidos de ámbito no estatal con un gran protagonismo y una diversidad de prioridades en las disputas electorales en los distintos niveles territoriales.

El objeto de análisis de este artículo hace referencia a los procesos de realineamiento electoral, pero en un contexto más amplio se vincula a la intensa emergencia de la cuestión identitaria (lo catalán vs. lo español). Así pues, lo que se estudia en este artículo puede clasificarse en los episodios del renacimiento de los nacionalismos periféricos (sin estado), como también ha sucedido en Escocia, Córcega, Quebec o el País Vasco. En un mundo globalizado, en un contexto de soberanías múltiples y compartidas, resurge el debate sobre el papel del Estado nación, las nuevas polaridades supra- y subestatales o la construcción de identidades naciones complejas. 


\section{La dinámica tradicional de la competencia electoral en Cataluña (1984-2010)}

El sistema de partidos en Cataluña gozó de casi tres décadas de estabilidad entre las elecciones de 1984 y las de 2010 (Castro, 2011; Sánchez Medero, 2011). Cinco son las características definitorias de los 26 años de estabilidad, desde la segunda convocatoria electoral al Parlament después de la recuperación democrática en 1984 hasta 2010. 1) La competición electoral se articuló a través de dos cleavages: el ideológico "izquierda-derecha" y el nacional "catalanismo-españolismo". La competencia dominante era en el eje izquierda-derecha (Barrio y Rodríguez, 2018) y adquiría una forma centrípeta. 2) El elevado número de partidos presentes en la cámara en todas las legislaturas, es decir, la fragmentación. Si bien en la mayoría de parlamentos autonómicos hasta 2015 lo habitual era la presencia de dos o tres partidos, en el legislativo de Cataluña siempre hubo al menos cinco grupos parlamentarios. 3) La centralidad que tuvo la federación de partidos que competía bajo las siglas de $\mathrm{CiU}$ que siempre ganó en escaños y en votos (menos en las elecciones de 1999 y 2003 en las que el PSC ganó en votos). 4) La abstención diferencial. Desde el restablecimiento del autogobierno en Cataluña hasta 2010 se registra una diferencia entre diez y veinte puntos porcentuales más de participación en las elecciones al Congreso de los Diputados que en las del Parlament de Cataluña. 5) Y complementaria a la anterior, el voto dual (Montero y Font, 1991), que se podía definir como "el comportamiento de una parte significativa del electorado que de una forma reiterada iba variando su apoyo entre las dos formaciones más influyentes del sistema, el PSC y CiU, en función del tipo de comicios" (Bartomeus, 2016, p. 91). (Ver Figura 1)

\section{¿Se puede hablar de realineamiento electoral en Cataluña? (2012-17)}

Las elecciones de diciembre 2012 marcan el inicio de un proceso de cambios. $\mathrm{Si}$ bien en aquella cita aún no se produjeron modificaciones sustantivas en el sistema de partidos, que no difiere mucho del de la época anterior, sí cambiaron ya los discursos, los cuales se enmarcaron en un frame en que la demanda de independencia tensionó el cleavage nacionalista (Vilaregut, 2018). Fue en este momento cuando los actores sociales que reivindicaban la independencia adquirieron un mayor protagonismo. Con ello, también cambiaron las prefe- 
Figura 1. Porcentaje de voto a los principales partidos en las elecciones al Parlament (1980-2010).

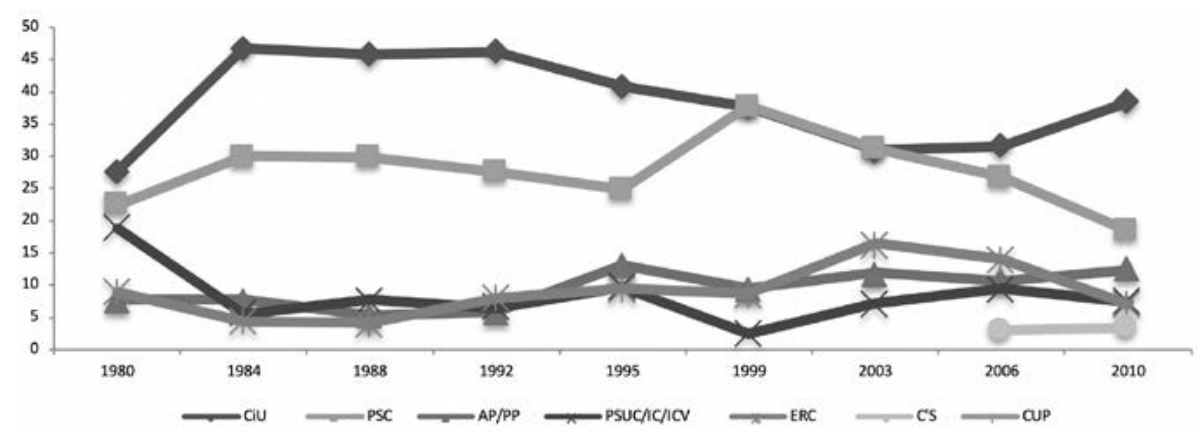

CiU: Convergència i Unió (centro derecha catalanista); PSC: Partit dels Socialistes de Catalunya (socialista); AP/PP: Alianza Popular/Partido Popular (derecha española); PSUC/ ICV: Partit Socialista Unificat de Catalunya/Iniciativa per Catalunya-Verds (ICV) (comunistas/ecosocialismo); ERC: Esquerra Republicana de Catalunya (izquierda independentista); C's: Ciudadanos (liberal anticatalanista); CUP: Candidatura d'Unitat Popular (anticapitalistas independentistas). Se dejan fuera las candidaturas que solo obtuvieron representación en una legislatura como Partido Socialista Andalucista (1980), Centro Democrático y Social (1988) y Solidaritat Catalana per la Independència (2010).

Fuente: Elaboración propia.

rencias de algunos partidos respecto de su política de pactos, como ERC que optó por apoyar a CiU. ${ }^{3}$ (Ver Tabla 1)

A continuación, y para validar la hipótesis presentada, en este apartado se examina si se dan los seis elementos que la literatura apunta a la hora de afirmar si existe o no un proceso de realineamiento electoral (Carreras, Morgestern y Su, 2015; Mayhew, 2002).

\section{Participación electoral excepcionalmente alta}

La participación electoral registrada en las elecciones al Parlament desde 1980 hasta 2010 siempre se mantuvo entre el 54 y el $65 \%$. Como se observa en la

3 Los resultados de ERC en las elecciones de 2010, después de la segunda experiencia del gobierno de izquierdas, fueron los peores en veinte años. En 2011, dimitió la dirección al completo y el congreso que le siguió (cabe señalar que los congresos de ERC no funcionan a través de delegados como en el resto de partidos sino de un modo asambleario) escogió a Oriol Junqueras, quien, a partir de entonces, lideró el partido sin oposición interna. 


\section{2 | Elecciones al Parlamento catalán 2012-2017: \\ Macià Serra Serra, Gemma Ubasart-González y Salvador Martí i Puig \\ ¿un proceso acelerado de realineamiento electoral?}

Tabla 1. Resultados de las elecciones al Parlament (2010-2017)

\begin{tabular}{ccccccccc}
\hline & 2010 & & 2012 & & 2015 & \multicolumn{2}{c}{2017} & \\
\hline & $\%$ votos & Escaños & $\%$ votos & Escaños & \% votos & Escaños & $\%$ votos & Escaños \\
\hline CiU/JxCat & 38,43 & 62 & 30,71 & 50 & & & 21,66 & 34 \\
\hline JxSí & & & & & 39,59 & 62 & & \\
\hline PSC & 18,38 & 28 & 14,43 & 20 & 12,72 & 16 & 13,86 & 17 \\
\hline PP & 12,37 & 18 & 12,98 & 19 & 8,49 & 11 & 4,46 & 4 \\
\hline $\begin{array}{c}\text { ICV-EUiA/ } \\
\text { CSQP/ } \\
\text { CeCP }\end{array}$ & 7,37 & 10 & 9,9 & 13 & 8,94 & 11 & 7,46 & 8 \\
\hline ERC & 7 & 10 & 13,7 & 21 & & & 21,38 & 32 \\
\hline SI & 3,29 & 4 & & & & & & \\
\hline C's & 3,39 & 3 & 7,57 & 9 & 17,9 & 25 & 25,35 & 36 \\
\hline CUP & & & 3,48 & 3 & 8,21 & 10 & 4,24 & 4 \\
\hline $\begin{array}{c}\text { Participa- } \\
\text { ción }\end{array}$ & 58,78 & 67,76 & & 74,95 & & 79,04 & \\
\hline
\end{tabular}

Fuente: Elaboración propia.

figura 2, en las elecciones al Congreso de los Diputados en Cataluña, la participación siempre fue superior. Sin embargo, en las tres últimas contiendas electorales autonómicas, la participación aumentó de forma intensa y acabó, a partir de 2015, con la abstención diferencial. Podría haberse de participación diferencial inversa: personas que normalmente no participaban de ningún proceso electoral, se movilizaron para acudir a las urnas. En este sentido, las elecciones al Parlament ya no se percibieron como elecciones de segundo orden. El debate sobre la independencia (o no) de Cataluña respecto del resto de España generó olas de movilización electoral sin precedentes, por lo que se cumplió con el primer elemento necesario para la existencia de un realineamiento electoral.

\section{Conflicto y discrecionalidad en la nominación de candidaturas: listas unitarias y peso de los independientes}

Hasta las elecciones de 2015 en todos los partidos con representación parlamentaria, con la excepción de algunas de formaciones minoritarias, como SI y la CUP, el líder del partido era quien encabezaba la candidatura electoral y, por 
Figura 2. Participación electoral en Cataluña en las elecciones generales y autonómicas.

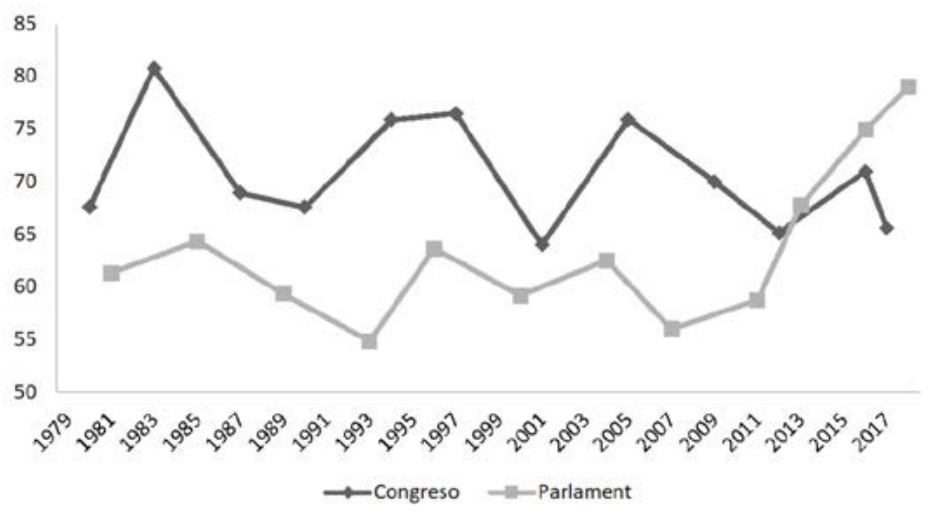

Fuente: Elaboración propia.

tanto, quien se presentaba ante los electores como candidato a la Presidencia de la Generalitat. En las elecciones de 2015, esta lógica cambió entre los partidos independentistas. Su intención de convertir las elecciones al Parlament en un plebiscito sobre la independencia tuvo consecuencias en las candidaturas presentadas y en la elaboración de las listas electorales. La candidatura de JxSí en 2015 se confeccionó con una lógica diferente de lo habitual hasta el momento. Los líderes de los partidos mayoritarios de la coalición (CDC y ERC) renunciaron a los primeros puestos en beneficio de otro tipo de liderazgos: Raül Romeva, que había sido diputado en el Parlamento Europeo por ICV, encabezó la lista en la circunscripción de Barcelona, ubicación tradicionalmente reservada al candidato a la Presidencia de la Generalitat. ${ }^{4}$ Le siguieron, en los números 2 y 3, respectivamente, Carme Forcadell y Muriel Casals, las líderes de la ANC y Ómnium Cultural, las entidades que habían convocado las movilizaciones independentistas en los últimos años. Estas decisiones desplazaron hasta los puestos 4 y 5 a los líderes de los partidos que impulsaban la coalición: Artur Mas por CDC y Oriol Junqueras por ERC, y posteriormente los dos partidos se repartieron los puestos en la lista conforme a su proporción de escaños en el Parlament.

Esta "nueva" lógica no solo se aplicó a la circunscripción de Barcelona, sino que también se llevó a cabo en las otras tres circunscripciones y dejó los dos pri-

4 En este sentido, durante la campaña electoral no quedo claro quién era realmente el candidato a la Presidencia de la Generalitat de la coalición. 


\section{4 | Elecciones al Parlamento catalán 2012-2017: \\ ¿un proceso acelerado de realineamiento electoral?}

meros puestos a personas sin vinculación orgánica con los partidos. A modo de ejemplo, en la circunscripción de Girona, encabezó la lista electoral el cantautor Lluís Llach, y el primer representante de un partido fue Carles Puigdemont quien ocupó el número 3 .

Pero la "anormalidad" no se redujo a las listas electorales. Siguió en la presentación de la candidatura a president de la Generalitat. Como JxSí no obtuvo la mayoría absoluta, buscó cuajar una mayoría parlamentaria con la CUP. Pero esta no quiso dar su apoyo en la investidura al president en funciones (Artur Mas). Después de agotar casi todo el periodo que la ley prevé entre la celebración de las elecciones y la elección de un jefe del Ejecutivo antes de que se vuelvan a convocar elecciones de nuevo, en el último momento JxSí propuso un candidato al que la CUP daría su apoyo: Carles Puigdemont. Si bien ya era alcalde de Girona y número 3 en la misma circunscripción, en ningún momento antes de las elecciones se intuyó que podría ser el escogido para president.

En las elecciones de 2017, la anormalidad en la nominación de candidatos se mantuvo en los principales partidos independentistas JxCat y ERC, que concurrieron por separado. En primer lugar, por la situación de sus líderes: Oriol Junqueras encabezó la candidatura de ERC estando en prisión provisional y Carles Puigdemont encabezó la candidatura de Junts per Catalunya (JxCat), nueva mutación de la antigua $\mathrm{CDC}$, aunque no pudo participar físicamente en la campaña electoral dado que se encontraba en Bélgica. Si bien el grueso de la candidatura estaba formada por miembros de PDeCAT, hubo muchos candidatos independientes que no fueron elegidos por los órganos de dirección de este, sino por Puigdemont. Por ejemplo, quienes encabezaron las listas en las circunscripciones de Girona y Lleida no tenían militancia orgánica y fueron designados debido a la confianza personal que tenían con el president depuesto. Todo ello contribuyó a la percepción de que la candidatura de JxCat no era exactamente la candidatura del PDeCAT.

La anormalidad volvió a aparecer en la investidura. Durante varias semanas, hubo intentos de investir a Carles Puigdemont primero y Jordi Sánchez después, candidatos que concurrieron como número 1 y número 2 , respectivamente, de JxCat. La mesa del Parlament no permitió que se celebrara el debate ni la votación de investidura, porque ambos candidatos no podían personarse en el hemiciclo. Después fue Jordi Turull quien sí se sometió a un proceso de investidura, pero perdió la primera votación y, posteriormente, fue encarcelado de manera preventiva. Al final, se barajaron numerosos candidatos (todos ellos de JxCat) sin importar el orden en que figuraban en las listas electorales. La persona que obtuvo el 
apoyo necesario del Parlament fue Joaquim Torra, quien figuraba de número once en las listas de JxCat en la circunscripción de Barcelona. Por tanto, la persona que se invistió como president de la Generalitat en la XIII legislatura tampoco lideró ninguna lista electoral.

Así pues, puede concluirse que tanto en las elecciones de 2015 como en las de 2017 los liderazgos públicos en las dos formaciones independentistas principales no solo cambiaron sino que se terminaron mutando de forma relativamente confusa (los cabezas de lista de 2015 de JxSí no eran los líderes orgánicos ni había certeza de que fueran realmente los candidatos a presidenciables), azarosa (la llegada a la Presidencia de la Generalitat de Puigdemont siendo el número 3 por la circunscripción de Girona o de Torra siendo el 11 por Barcelona) y discrecional (ya que la lista de JxCat fue escogida por Puigdemont más que por el PDeCAT).

Con ello, se hace evidente que desde 2015 ha reinado en las formaciones mayoritarias del independentismo catalán una lógica confusa en la elaboración de listas y en la presentación de candidaturas para jefe del Ejecutivo.

\section{Conflicto y discrecionalidad en la nominación de candidaturas: listas unitarias y peso de los independientes}

El sistema de partidos en Cataluña ha cambiado en el lustro 2012-2017. Han desaparecido partidos, han aparecido otros nuevos y ha aumentado la fragmentación. Durante el largo periodo de estabilidad, casi siempre obtenían representación los mismos cinco partidos. Los dos grandes partidos, CiU y PSC, sumaban de media más de $2 / 3$ de votos. En las elecciones de 2012, este apoyo empezó a flaquear y por primera vez no llegaron a la mitad de los sufragios $(45,14 \%)$. En las últimas elecciones, la suma de los herederos de CDC (JxCat), del PSC y de UDC solo consiguieron el 35,52 \% de los votos. Ello da cuenta de que los grandes partidos de antańo perdieron aproximadamente la mitad de la fuerza que habían mantenido desde el inicio de la democracia.

Como muestra la figura 3, han aparecido dos nuevos partidos y otros dos han cambiado tanto que ya casi no se parecen a lo que solían ser, hasta dibujar un sistema de siete partidos en el que ninguno de ellos puede gobernar en solitario. 


\section{6 | Macià Serra Serra, Gemma Ubasart-González y \\ ¿un proceso acelerado de realineamiento electoral?}

El que ha obtenido un mayor éxito en los últimos años es Ciudadanos. El partido se fundó y se presentó por primera vez a las elecciones de 2006, en que obtuvo poco más del $3 \%$ de votos en la circunscripción de Barcelona y 3 escaños. En las siguientes elecciones, revalidó el resultado y ejerció un papel testimonial e impugnador del "consenso catalanista" que incluía el catalán como lengua vehicular en la escuela, la ley de política lingüística o los medios de comunicación públicos en catalán. Aquel consenso era asumido por todos los partidos de la cámara excepto el PP.

No obstante su oposición frontal a la dinámica independentista del Gobierno catalán y el desgaste de un PP que aplicó políticas de austeridad desde el Gobierno de España y al que afectaron numerosos casos de corrupción, catapultaron el nuevo partido desde los tres escaños en 2006 y 2010 a 9 en las elecciones de 2012, 27 en 2015, hasta convertirse en el primer partido en el Legislativo catalán en 2017 con 36 escaños. Este crecimiento continuado, junto con otros factores, contribuyó a que el partido dejara de ser de ámbito exclusivamente catalán e irrumpiera también en las Cortes Generales en las elecciones del 2015 (Rodríguez y Barrio, 2016).

Figura 3. Las principales coaliciones de partidos en las tres últimas elecciones al Parlament.

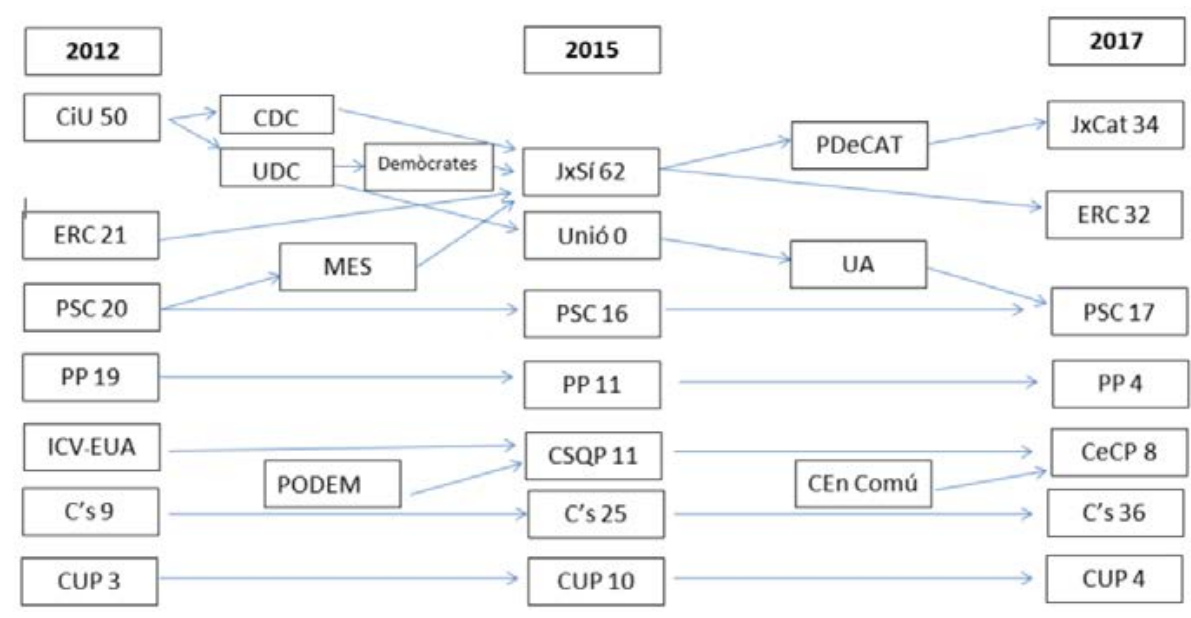

Fuente: Elaboración propia. 
En 2010, obtuvo representación en el Parlament el partido Solidaritat per la Independencia (SI) con cuatro escaños, pero en las siguientes elecciones desapareció. En 2012, un nuevo partido independentista, la Candidatura d'Unitat Popular (CUP), obtuvo representación y la ha seguido manteniendo hasta el día de hoy. Aunque ha obtenido resultados dispares (tres, diez y cuatro escaños), su influencia ha sido considerable dado que la mayoría gubernamental ha buscado su apoyo en el Parlament desde 2015.

Además de los partidos estrictamente nuevos, ha habido otras dos formaciones políticas que han cambiado de nombre y han sufrido transformaciones profundas. La coalición CiU, que había sido la primera fuerza en escaños en todas las elecciones en el Parlament, desapareció. Uno de los partidos que la formaban, CDC, se transformó en PDeCAT, pero se acabó presentando a las elecciones como JxCat. En este proceso, radicalizó su postura a favor de la independencia al tiempo que desdibujó sus rasgos ideológicos y se afirmó como un proyecto transversal. Actualmente, existen tres organizaciones en este espacio político: PDeCAT, JuntsxCat y Crida. Algunos antiguos dirigentes de CiU crearon otras formaciones políticas ${ }^{5}$ sin demasiado éxito hasta el momento.

Otro cambio sustancial fue el de ICV-EUiA. En 2015, la coalición llegó a acuerdos para articularse electoralmente con Podem (organización catalana de Podemos) y Barcelona en Comú (partido de la alcaldesa de Barcelona) y se presentó a las elecciones con la candidatura Catalunya Sí Que Es Pot. El 8 de abril de 2017, se realiza la asamblea constituyente de Catalunya en Comú que pretende unificar bajo un mismo paraguas organizaciones políticas y sociales de la izquierda catalana (Podem en último momento decide quedarse al margen de la nueva formación). Las elecciones de 2017 son las primeras en las que la organización concurre como tal, en coalición con la formación morada. Si bien los resultados electorales en las elecciones al Parlament han sido similares en votos y escaños a los cosechados por la antigua ICV-EUiA, ha sido en las elecciones municipales y generales cuando han conseguido los mejores resultados, como la primera plaza en las elecciones municipales en Barcelona de 2015 o en las elecciones generales en Cataluña en 2015 y 2016.

5 Como Antoni Fernández Teixidor que fundó Lliures o Ramon Espadaler que fundó Units per Avançar. 


\section{8 | Elecciones al Parlamento catalán 2012-2017: \\ ¿un proceso acelerado de realineamiento electoral?}

Otro dato importante es el aumento de la fragmentación que se produce en este último periodo como puede verse en la figura 4. Aun así, cabe señalar que la disminución del indicador en 2015 es debida a JxSí, que aúna lo que en las otras convocatorias son dos candidaturas separadas.

Figura 4. Número efectivo de partidos por legislatura.

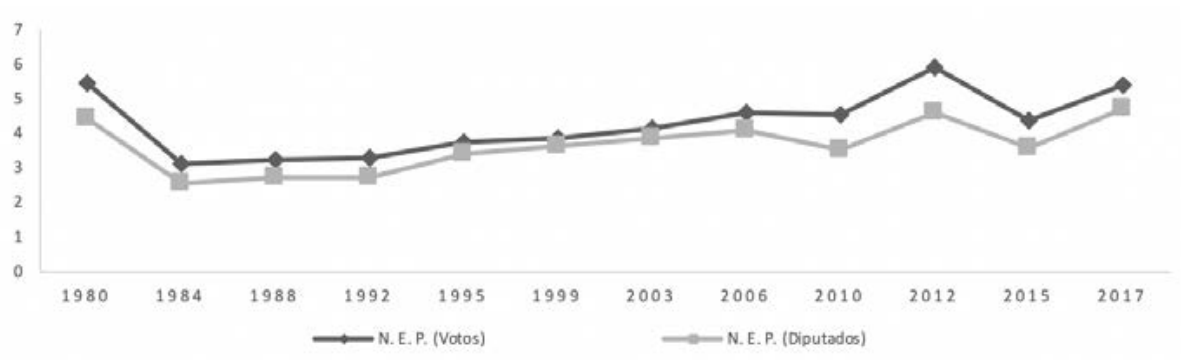

Fuente: Elaboración propia.

Así las cosas, en Cataluña, se pasó de un sistema estable de cinco partidos con dos grandes y tres pequeños a un sistema mucho más fragmentado, con siete partidos sin ninguno preeminente y con apenas cuatro puntos porcentuales de diferencia entre el primer partido y el tercero.

\section{La aparición de nuevos temas de conflicto (issue cleavages): la cuestión nacional en el centro}

Como muestra la figura 5, después del impacto de la crisis económica en Europa y hasta 2017, el principal problema para la mayoría de catalanes era el paro y la precariedad laboral. Pero, desde 2011, las relaciones Cataluña-España fueron abriéndose hueco entre un número de personas cada vez mayor, hasta alcanzar la primera posición. Paralelamente, también ha crecido la insatisfacción con la política.

Pero desde mucho antes que las relaciones Cataluña-España fueran el mayor problema para los ciudadanos, ya lo eran para los partidos políticos, que, desde 2012, construían las coaliciones de gobierno a partir de esta cuestión.

Como ya se ha comentado, el independentismo no es una propuesta política nueva en Cataluña, sin embargo, nunca había sido central (Amat, 2017). Desde 
Figura 5. Principal problema de Cataluña.



Nota: Es una pregunta abierta, por lo que hay muchas respuestas posibles, por lo que solo se presentan las tres con mayor porcentaje actualmente.

Fuente: Elaboración propia a partir de datos de los barómetros del CEO.

la década de 1980, las principales reivindicaciones se centraban en las demandas de mayor autogobierno o reconocimiento de la identidad, que introdujeron la idea de un marco de relación federal o plurinacional con el Estado. Lo nuevo en este último lustro ha sido la emergencia del independentismo o secesionismo como elemento central en el debate público en Cataluña. En la década de 1980, ningún partido con representación parlamentaria defendía la independencia de Cataluña. No fue hasta 1989 que ERC abrazó la idea. Desde entonces, esta formación obtuvo entre el 7 y el $16 \%$ de los sufragios en las elecciones autonómicas. En 2010, un nuevo partido que impulsó la demanda independentista, no como utopía lejana sino como su razón de ser, fue SI. En 2012, se le sumó la CUP, aunque por aquel entonces tenía mayor ascendencia su corriente anticapitalista que la independentista. Después de 2012, se registró la conversión de CDC del catalanismo al independentismo. Desde entonces, en el Parlament hay una mayoría para la cual la secesión de Cataluña (más allá de su autoposicionamiento ideológico) es su hito, y a él subsume el resto de su agenda.

Una consecuencia derivada de que el debate acerca de la independencia se haya erigido como principal tema de la agenda política en Cataluña es que el debate alrededor de las políticas públicas ha quedado en un segundo plano desde 2012. En los debates parlamentarios y en los manifiestos partidarios, ha habido 


\section{$\mathbf{1 2 0}$ | Macià Serra Serra, Gemma Ubasart-González \\ ¿un proceso acelerado de realineamiento electoral?}

una escasa mención a las policies, pues el debate se ha centrado en la polity. En este sentido, los cambios que se han propuesto (o que se han combatido) están relacionados con la definición del demos y de los derechos de ciudadanía más que con las diversas políticas públicas sectoriales.

Quizás una de las consecuencias de los cambios producidos es la configuración de mayorías parlamentarias coherentes en relación con la independencia, pero que no están de acuerdo con un grueso importante de políticas públicas. Una muestra de ello es que desde las elecciones catalanas de 2012 y hasta las elecciones generales de 2019 solo se hayan aprobado tres presupuestos de la Generalitat. ${ }^{6}$

\section{Una fuerte polarización}

La polarización, la distancia ideológica entre los partidos, ha aumentado durante el periodo analizado en el sistema de partidos en Cataluña. El grado de polarización se calcula a partir del autoposicionamiento de los individuos y de la percepción que estos tienen de la posición que ocupan las diversas fuerzas políticas, en relación con los clivajes que fracturan la sociedad: en nuestro caso el eje derecha-izquierda y el nacional. Barrio y Rodríguez (2018, p. 232), utilizando la fórmula de Rusell Dalton, constatan que hasta 2010 la polarización de votantes no superaba el 3 en el eje ideológico, ni el 3,5 en el eje nacional, en una escala de 0 a 10. La distancia entre partidos era un poco superior, aunque no superaba el 4 en ninguno de los dos casos. A partir de 2010, los índices que hacen referencia al eje nacional se disparan situándose en 2015 en torno a 5,5 si se toma en consideración al votante y un 6,5 si se hace con los partidos.

Siguiendo en esta lógica de polarización, hoy el sistema político de Cataluña se organiza en torno a dos bloques de partidos: el independentista y el unionista. La dinámica de competencia ha pasado de ser centrípeta, en competencia por el centro político, a centrífuga, en que mayoritariamente se compite con otros partidos en el mismo espacio, independentista o unionista. Este desplazamiento "radicalizante" se produce, sobre todo, por el papel importante que adquieren dos fuerzas políticas relativamente nuevas situadas en posiciones extremas: CUP

6 Los presupuestos de 2014 (Ley 1/2014, de 24 de junio, de Transparencia Pública de Andalucía.), 2015 (Ley 2/2015, de 30 de marzo, de desindexación de la economía española) y 2017 (Ley 4/2018, de 11 de junio, por la que se modifica el texto refundido de la Ley General para la Defensa de los Consumidores y Usuarios y otras leyes complementarias, aprobado por Real Decreto Legislativo 1/2007, de 16 de noviembre). 
y Ciudadanos. El partido que se sitúa a medio camino, con identidades plurales en su interior (Catalunya en Comú), aceptando la posibilidad de un referéndum de independencia si bien no estando a favor de esta, le cuesta articularse y obtener buenos resultados en un entorno tan tensionado por la cuestión nacional.

Barrio y Rodríguez (2018, p. 233) recuerdan otro dato importante: la polarización en el eje ideológico en Cataluña se ha situado en niveles inferiores respecto de lo sucedido en el sistema de partidos políticos español. Las tensiones experimentadas en los sistemas políticos en los diferentes contextos europeos fruto de la crisis económica y de la gestión neoliberal de esta han tenido efectos diversos en cada sistema de partidos. Así pues, por ejemplo, mientras que en el caso catalán la polarización se produce en el eje nacional, en el caso espańol se concentra en el eje ideológico.

\section{Aumento de la volatilidad electoral}

La volatilidad, el porcentaje de electores que cambian de voto de una elección a otra, es otro elemento clave para determinar si ha habido un realineamiento electoral. El índice de volatilidad de Peterson ${ }^{7}$ en las ocho últimas elecciones al Parlament. La volatilidad se mantuvo estable hasta las elecciones de 2012, la cual osciló entre el 7,4 y el 16,1\%. Pero, en las elecciones de 2015, se disparó hasta el $57,8 \%$. Más de la mitad de los electores cambiaron su voto de las elecciones de 2012 a las de 2015. Esta cifra es muy relevante y es una muestra de la alteración que ha sufrido el patrón de comportamiento electoral en Cataluña. Buena parte de esta volatilidad se explica por la irrupción de la candidatura de JxSí, formada por CDC y ERC, entre otros, ya que todos sus votos sumaron para la volatilidad, dado que era una candidatura nueva. El mismo fenómeno sucedió a la inversa cuando se disolvió en 2017. Pero la irrupción de JxSí solo explica parte del aumento de la volatilidad. Si se comparan las elecciones de 2012 y 2017 , el índice se eleva hasta el $27 \%$, también muy superior a la media que hubo entre 1995 y 2012.

Pero esta volatilidad no indica que los cambios se hayan producido en todas las direcciones. La volatilidad se ha producido dentro de los bloques a favor y en contra de la independencia de Cataluña. Muestra de ello es que, pese a la alta vo-

7 El índice de volatilidad electoral de Pedersen (Pedersen, 1979) se obtiene al sumar todas las diferencias en el porcentaje de voto de las distintas candidaturas y dividirlo por la mitad. El resultado muestra el porcentaje de electores que cambiaron su voto de una elección a otra. Por ejemplo, el índice de volatilidad de 2017 es la diferencia entre las elecciones de 2015 y 2017. 


\section{2 | Macià Serra Serra, Gemma Ubasart-González \\ ¿un proceso acelerado de realineamiento electoral?}

latilidad entre las elecciones de 2015 y 2017, apenas varió la suma del porcentaje de voto a los partidos independentistas, del 47,8 \% en 2015 al 47,5\% en 2017.

\section{Conclusiones}

Después de desgranar los diversos supuestos que dan cuenta de la existencia o no de un realineamiento en el sistema de partidos en Cataluña, se puede validar la hipótesis de que sí ha habido realineamiento, y de forma rápida, en el lustro 2012-2017. La centralidad que ha adquirido en este periodo el cleavage nacional ha generado un terremoto en el sistema de partidos y en el ámbito electoral. Si bien es cierto que la cuestión nacional ha estado presente en la vida política catalana desde la segunda mitad del siglo XIX (momento en que se encuentran los primeros pasos del catalanismo), esta cuestión no era la central a la hora de guiar el voto. En este marco, se ha incrementado — en el inicio del ciclo- la volatilidad electoral y se ha experimentado un aumento sostenido de la participación de un electorado tradicionalmente poco activo en los comicios autonómicos. Con ello, han aparecido nuevas formaciones con representación parlamentaria y se han incrementado la polarización y la incertidumbre a la hora de elegir los candidatos, tanto en las cabeceras electorales como en los puestos de máxima responsabilidad. Fruto de todo ello puede afirmarse que se han consolidado nuevas pautas de comportamiento electoral que a la vez han puesto las bases para la transformación del sistema de partidos con cambios muy relevantes respecto de la etapa anterior, tanto en lo relativo a su número como a su composición y propuesta política. Algunos partidos han emergido para quedarse (Cs o) en el mapa político catalán y otras formaciones han mutado significativamente como ICV-EUiA que se ha transformado en un espacio más amplio que toma por nombre Catalunya en Comú (coaligado a Podemos), o CDC que se ha venido refundando desde que abrazó los postulados independentistas en diversas formaciones y plataformas electorales. Sin embargo, este proceso no es estático sino fluido y en constante interacción con el sistema de partidos español que, a su vez, está experimentando también transformaciones profundas.

\section{Referencias}

Amat, J. (2017). Largo proceso, amargo sueño: cultura y política en la Cataluña contemporánea. Barcelona, España: Tusquets.

Baras, M. y Matas, J. (1998). Els partits polítics i el sistema de partits. En M. Caminal Badia y J. Matas Dalmases (Eds.), El sistema polític de Catalunya. Madrid, España: Tecnos. Pp.: 213-228. 

¿un proceso acelerado de realineamiento electoral?

Barrio, A. y Rodríguez-Teruel, J. (2018). El sistema de partits. En G. Ubasart-González y S. Martí (Eds.), Política i govern a Catalunya: de la transicio' a l'actualitat. Madrid, España: Catarata. Pp.: 217-243.

Bartomeus O. (2016). La influència del relleu generacional en la transformació del comportament electoral a Catalunya (Tesis doctoral, Universitat Autònoma de BarceIona, Barcelona, España). Recuperado de https://www.tdx.cat/bitstream/handle/10803/399560/obb1de1.pdf?sequence=12isAllowed =y

Carreras, M., Morgenstern, S. \& Su, Y. P. (2015). Refining the theory of partisan alignments: evidence from Latin America. Party Politics, 21(5), 671-685.

Castro, C. (2011). Las claves de las elecciones autonómicas catalanes (1980-2010). En J. Marcet y X. Casals (Eds.), Partidos y elecciones en la Cataluña del siglo XXI. Barcelona, España: Institut de Ciències Polítiques i Socials. Pp.: 13-48.

Fernández-Albertos, J. (2018). Anti-sistema: desigualdad económica y precariado político. Madrid, España: Catarata.

Guibernau, M. (2004). Catalan nationalism: Francoism, transition and democracy. Londres, RU: Routledge.

Hernández, E. \& Kriesi, H. (2016). The electoral consequences of the financial and economic crisis in Europe. European Journal of Political Research, 55(2), 203-224.

Institut de Ciències Polítiques i Socials. (2012). Anuari polític de Catalunya 2011. Barcelona, España: Autor.

Key Jr, V. O. (1955). A theory of critical elections. The Journal of Politics, 17(1), 3-18.

Key Jr, V. O. (1959). Secular realignment and the party system. The Journal of Politics, 21(2), 198-210.

Key Jr, V. O. (1964). The party battle, 1896-1969. En Autor, Politics, parties, and pressure groups. Nueva York, EE. UU.: Thomas Y. Crowell Company. Pp.: 151-177.

Kriesi, H. \& Pappas, T. (2015). European populism in the shadow of great recession. Colchester, RU: European Journal of Political Research.

Ley 1/2014, de 24 de junio, de Transparencia Pública de Andalucía. BOE, núm. 172 (2014).

Ley 10/2014, de 26 de septiembre, de consultas populares no referendarias y otras formas de participación ciudadana. BOE, núm. 64 (2014).

Ley $2 / 2015$, de 30 de marzo, de desindexación de la economía española. $B O E$, núm. 77 (2015).

Ley 4/2018, de 11 de junio, por la que se modifica el texto refundido de la Ley General para la Defensa de los Consumidores y Usuarios y otras leyes complementarias, aprobado por Real Decreto Legislativo 1/2007, de 16 de noviembre. BOE, núm. 142 (2018).

Marcet, J. (2011). Las relaciones entre el sistema de partidos de Cataluña y España y la articulación partidària. En J. Marcet y X. Casals (Eds.), Partidos y elecciones en la Cataluña del siglo XXI. Barcelona, España: Institut de Ciències Polítiques i Socials. Pp.: 191-232.

Mayhew, D. (2002). Electoral realignments: A critique of an American genre. New Haven, EE. UU.: Yale University Press.

Molas, I. y Bartomeus, O. (1998). Estructura de la competència política a Catalunya. Barcelona, España: Institut de Ciències Polítiques i Socials.

Pedersen, M. N. (1979). The dynamics of European party systems: Changing patterns of electoral volatility. European Journal of Political Research, 7(1), 1-26. 
Macià Serra Serra, Gemma Ubasart-González y Salvador Martí i Puig

124 | Elecciones al Parlamento catalán 2012-2017:

¿un proceso acelerado de realineamiento electoral?

Sánchez Medero, G. (2011). El sistema de partidos en Cataluña (1980-2010). Revista de Derecho Electoral, 12, 1-34.

Ubasart-González, G. (2020). The independence procés: the triple Spanish crisis and an unresolved question of sovereignty. En García Agustin, Oscar and Malayna Raftopoulos, Nations at stake. The Catalan march for independence, Palgrave (in press).

Vilaregut, R. (2018). El moviment independentista a Catalunya. En G. Ubasart-González y S. Martí i Puig (Coords.), Política i govern a Catalunya: de la transicio' a l'actualitat. (pp. 325-342). Madrid, España: Catarata. 